\title{
Observation of clinical efficacy and toxic and side effects of pirarubicin combined with cytarabine on acute myeloid leukemia
}

\author{
SUQIN LV ${ }^{1}$, AIHUA LI ${ }^{2}$, HAIJUAN WU ${ }^{2}$ and XIAOLI WANG ${ }^{3}$ \\ ${ }^{1}$ Outpatient Pharmacy, Departments of ${ }^{2}$ Pharmacy and ${ }^{3}$ Radiotherapy, \\ Weifang People's Hospital, Weifang, Shandong 261041, P.R. China
}

Received September 28, 2018; Accepted January 8, 2019

DOI: 10.3892/ol.2019.9966

\begin{abstract}
Efficacy and toxic and side effects of pirarubicin combined with cytarabine and mitoxantrone combined with cytarabine on the treatment of initially treated acute myeloid leukemia (AML) were compared. A retrospective analysis was performed on the medical records of $76 \mathrm{AML}$ patients who were initially treated in Weifang People's Hospital. Among them, 36 patients (observation group) were treated with pirarubicin combined with cytarabine, and 40 patients (control group) were treated with mitoxantrone combined with cytarabine. The efficacy and toxic and side effects on patients in the two groups were observed. There was no statistically significant difference in the complete response (CR) rate, partial response (PR) rate and overall response (OR) rate of patients between the two groups $(\mathrm{P}>0.05)$. Patients in the observation group had significantly lower incidence of cardiotoxicity and alopecia than those in the control group $(\mathrm{P}<0.05)$. Patients in the observation group had lower incidence of bone marrow depression (BMD) at grade IV than those in the control group $(\mathrm{P}<0.05)$. The median progression-free survival time of patients was 14.5 months in the observation group and 18 months in the control group. The progression-free survival rate of patients was $36.11 \%$ in the observation group and $40.00 \%$ in the control group, with no difference between the two groups $(\mathrm{P}>0.05)$. The median survival time of patients was 22.5 months in the observation group and 24.5 months in the control group. The overall survival (OS) rate of patients was $44.44 \%$ in the observation group and $47.50 \%$ in the control group, with no difference between the two groups $(\mathrm{P}>0.05)$. Both pirarubicin combined with cytarabine and mitoxantrone combined with cytarabine have satisfactory efficacy on
\end{abstract}

Correspondence to: Dr Haijuan Wu, Department of Pharmacy, Weifang People's Hospital, 151 Guangwen Road, Weifang, Shandong 261041, P.R. China

E-mail: hyc6u5@163.com

Key words: pirarubicin, cytarabine, acute myeloid leukemia, toxic and side effects initially treated AML. Compared to the latter, the former has lower toxic and side effects.

\section{Introduction}

Acute myeloid leukemia (AML), a fast-growing and fatal hematological malignant tumor, is the most common in adult leukemia. Its annual incidence is 3-4 patients per 100,000 people and its 5 -year mortality is $75 \%$. The incidence of its onset is increasing with age (1-3). Myeloid archaeocytes clonally proliferate in peripheral blood or bone marrow, which is the characteristic of AML (4). At present, AML is commonly treated by multi-drug combined with chemotherapy, and a study confirmed that it may be cured after treated by multi-drug combined with chemotherapy (5). However, platelet and other indicators in some AML patients have not been completely recovered or retained some minor lesions after inductive treatment, so they have not achieved complete response (CR), with a great possibility of recurrence (6). Therefore, the availability of $\mathrm{CR}$ is the key to the prognostic survival of AML patients.

The most classic regimen for AML in clinical practice today is daunorubicin combined with cytarabine, but this regime has been not changed in the past few decades, so AML has certain resistance to it (7). The most important thing is that daunorubicin, an anthracycline that improves the efficacy in treating leukemia, is often accompanied by greater cardiotoxicity (8) to which patients are intolerant in the long term. In recent years, many combination regimens that consist of new drugs have attracted the attention of a wide range of scholars. Among them, as a new generation of anthracycline anticancer drugs, pirarubicin has better activity in various multidrug-resistant malignant tumors, and exerts an anti-tumor effect with smaller toxic and side effects when used alone or in combination with other drugs (9). Mitoxantrone is a new type of anti-tumor drugs and has better efficacy in treating breast cancer, leukemia and lymphoma. Besides, the cardiotoxicity caused by it is lower than that caused by similar drugs at the same clinical dose (10). Existing studies have shown that both pirarubicin combined with cytarabine and mitoxantrone combined with cytarabine have the same efficacy as the traditional daunorubicin regimen in the treatment of acute leukemia, which have smaller cardiac toxic and side effects, and generally better tolerance 
in patients $(11,12)$. However, there are currently few studies on the comparison of efficacy and safety between pirarubicin and mitoxantrone in clinical practice. Therefore, in this study, the efficacy and toxic and side effects of these two drugs in combination with cytarabine on the treatment of AML were compared in detail, in order to provide a clinical reference for the treatment of AML patients.

\section{Materials and methods}

Patient information. A total of 76 AML patients who were initially treated in Weifang People's Hospital (Weifang, China) were collected. Among them, 36 patients were treated with pirarubicin combined with cytarabine as the observation group, and 40 patients were treated with mitoxantrone combined with cytarabine as the control group. Patients in the observation group included 24 males and 12 females, aged 22-61 years, with an average age of $43.75 \pm 6.78$ years. Patients in the control group included 27 males and 13 females, aged 21-63 years, with an average age of $42.53 \pm 7.42$ years. Based on the FAB classification (13), there were $5 \mathrm{M}_{1}$ patients, $19 \mathrm{M}_{2}$ patients, $3 \mathrm{M}_{4}$ patients and $9 \mathrm{M}_{5}$ patients in the observation group. $5 \mathrm{M}_{1}$ patients, $22 \mathrm{M}_{2}$ patients, $4 \mathrm{M}_{4}$ patients and $9 \mathrm{M}_{5}$ patients in the control group. Based on the evaluation criteria for chromosome karyotype (14), all patients were grouped with genetic prognostic risk. There was 1 patient with high risk, 30 patients with medium risk and 5 patients with low risk in the observation group. Two patients with high risk, 36 patients with medium risk and 2 patients with low risk in the control group (Table I).

Inclusion criteria: i) All patients who met the diagnostic criteria for AML (15); and ii) patients who were initially diagnosed with AML, and were older than 18 years.

Exclusion criteria: i) Patients complicated with multiple organ dysfunction syndrome or other related leukemia such as acute and chronic lymphocytic leukemia; ii) patients complicated with severe cardiopulmonary insufficiency and liver and kidney dysfunction or (with) severe coagulation disorder; and iii) pregnant and lactating females.

All the contents of this study were approved by the Medical Ethics Committee of Weifang People's Hospital. Patients who participated in this research had complete clinical data. The signed informed consents were obtained from the patients or the guardians.

Treatment methods. Patients in the observation group were treated with pirarubicin combined with cytarabine. Pirarubicin (H10930105; Shenzhen Wanle Pharmaceutical Co., Ltd., Shenzhen, China) $25 \mathrm{mg} / \mathrm{m}^{2}$ was intravenously dripped by surface area from d1 to d3; cytarabine (H20055127; Zhejiang Yixin Pharmaceutical Co., Ltd., Zhejiang, China) $100 \mathrm{mg} / \mathrm{m}^{2}$ was intravenously dripped by surface area from 11 to $\mathrm{d} 7$. Patients in the control group were treated with mitoxantrone combined with cytarabine. Mitoxantrone (H10960190; Sichuan Shenghe Pharmaceutical Co., Ltd., Chengdu, China) $10 \mathrm{mg} / \mathrm{m}^{2}$ was intravenously dripped by surface area from d1 to d3; cytarabine $100 \mathrm{mg} / \mathrm{m}^{2}$ was intravenously dripped by surface area from d1 to d7. During the treatment, all patients were given liver protection and anti-emesis treatment. Antibiotics treatment was given to them in time and fluid infusion supportive treatment was strengthened if there was a co-infection.
Granulocyte colony-stimulating factor $300 \mu \mathrm{g} /$ day was used when patients' leukocytes were $<1.0 \times 10^{9} / 1$ or neutrophils were $<0.5 \times 10^{9} / 1$, until their leukocytes were $>2.0 \times 10^{9} / 1$. Apheresis platelet $8 \mathrm{U}$ was infused every 2 days when patients' platelets were $<20 \times 10^{9} / 1$, and erythrocytes were infused when their hemoglobin was $<6 \mathrm{~g} / \mathrm{l}$. One week was one treatment course in the observation and the control group, and the next treatment course was performed every 4 weeks.

Outcome measures. During the treatment, patients' symptoms and signs were closely observed. The blood routine of patients in the observation and the control group was reexamined twice a week, and once a week after their symptoms were relieved. Their routine electrocardiogram, liver function and renal function were examined within 1 week after the treatment. Their bone marrow morphology was examined at 2-3 weeks after the treatment, and once a month after their symptoms were relieved. According to the efficacy evaluation criteria (14), the efficacy was divided into CR and partial response (PR), and the overall response (OR) rate of the treatment of patients was the sum of CR and PR. The efficacy was systematically evaluated after 2 treatment courses. Consolidation therapy was performed on patients with $\mathrm{CR}$, and other regimens for treatment were performed on patients with PR. The consolidation therapy was performed based on a high-dose of cytarabine for 1 year. The follow-up was performed on patients after discharge when 1 treament course was completed or those who died halfway to calculate the OR rate. The National Cancer Institute Common Toxicity Criteria (NCI-CTC.4.0) of USA (15) was used for the comprehensive evaluation of safety.

Follow-up methods. Patients in the observation and the control group were followed up until they died or withdrew from the experiment. Telephone follow-up was performed on the 15th of each month in order to ask their quality of life and survival in detail.

Statistical analysis. SPSS19.0 statistical software (SPSS Inc., Chicago, IL, USA) was used to statistically analyze the experimental data, and GraphPad Prism 7 (Beijing Huanzhong Ruichi Technology Co., Ltd., Beijing, China) to plot the figures. Enumeration data were expressed as $\%$, and $\chi^{2}$ test was used for comparison between groups. Measurement data were expressed as (mean $\pm \mathrm{SD}$ ), and independent sample t-test was used for comparison between groups. Kaplan-Meier was used for survival analysis, with log-rank test. $\mathrm{P}<0.05$, indicates the difference is statistically significant.

\section{Results}

Comparison of general information. There were no statistically significant differences in the age, sex composition, clinical manifestations, clinical classification and blood routine of patients between the two groups $(\mathrm{P}>0.05)$ (Table I).

Comparison of efficacy. The efficacy on patients in the observation and the control group was compared. The results showed that the $\mathrm{CR}, \mathrm{PR}$ and $\mathrm{OR}$ rates of patients in the observation group were $80.56,5.56$ and $86.11 \%$, respectively, and those in the control group were 75.00 , 
Table I. Comparison of general information between observation and control group (mean \pm SD) [n (\%)].

\begin{tabular}{|c|c|c|c|c|}
\hline \multirow[b]{2}{*}{ Clinical factors } & \multicolumn{2}{|c|}{ Groups } & \multirow[b]{2}{*}{$t / \chi^{2}$} & \multirow[b]{2}{*}{ P-value } \\
\hline & Observation $(\mathrm{n}=36)$ & Control $(n=40)$ & & \\
\hline Age & $43.75 \pm 6.78$ & $42.53 \pm 7.42$ & 0.749 & 0.456 \\
\hline Body mass index $\left(\mathrm{kg} / \mathrm{m}^{2}\right)$ & $21.38 \pm 3.19$ & $22.13 \pm 2.83$ & 1.079 & 0.284 \\
\hline Sex & & & 0.006 & 0.939 \\
\hline Male & $24(66.67)$ & $27(67.50)$ & & \\
\hline Female & $12(33.33)$ & $13(32.50)$ & & \\
\hline Age & & & 0.023 & 0.879 \\
\hline$\leq 50$ years & $24(66.67)$ & $26(65.00)$ & & \\
\hline$>50$ years & $12(33.33)$ & $14(35.00)$ & & \\
\hline Clinical manifestations & & & 0.423 & 0.809 \\
\hline Fever & $15(41.67)$ & $16(40.00)$ & & \\
\hline Hepatosplenomegaly & $13(36.11)$ & $17(42.50)$ & & \\
\hline Others & $8(22.22)$ & $7(17.50)$ & & \\
\hline FAB classification & & & 0.152 & 0.985 \\
\hline $\mathrm{M}_{1}$ & $5(13.89)$ & $5(12.50)$ & & \\
\hline $\mathrm{M}_{2}$ & $19(52.78)$ & $22(55.00)$ & & \\
\hline $\mathrm{M}_{4}$ & $3(8.33)$ & $4(10.00)$ & & \\
\hline $\mathrm{M}_{5}$ & $9(25.00)$ & $9(22.50)$ & & \\
\hline Leukocytes before treatment & & & 2.129 & 0.145 \\
\hline$\leq 50 \times 10^{9} / 1$ & $28(77.78)$ & $36(90.00)$ & & \\
\hline$>50 \times 10^{9} / 1$ & $8(22.22)$ & $4(10.00)$ & & \\
\hline Hemoglobin before treatment & & & 0.097 & 0.755 \\
\hline$\leq 90 \mathrm{~g} / 1$ & $24(66.67)$ & $28(70.00)$ & & \\
\hline$>90 \mathrm{~g} / 1$ & $12(33.33)$ & $12(30.00)$ & & \\
\hline Platelets before treatment & & & 0.121 & 0.728 \\
\hline$\leq 40 \times 10^{9} / 1$ & $23(63.89)$ & $24(60.00)$ & & \\
\hline$>40 \times 10^{9} / 1$ & $13(36.11)$ & $16(40.00)$ & & \\
\hline Proportion of bone marrow blast cells & & & 0.029 & 0.864 \\
\hline$\leq 50 \%$ & $16(44.44)$ & $17(42.50)$ & & \\
\hline$>50 \%$ & $20(55.56)$ & $23(57.50)$ & & \\
\hline Risk stratification & & & 3.800 & 0.150 \\
\hline Low risk & $22(61.11)$ & $16(40.00)$ & & \\
\hline Middle risk & $8(22.22)$ & $11(27.50)$ & & \\
\hline High risk & $6(16.67)$ & $13(32.50)$ & & \\
\hline
\end{tabular}

10.00 and $85.00 \%$, respectively. There were no statistically significant differences in the CR, PR and OR rates of patients between the two groups $(\mathrm{P}>0.05)$ (Table II). The $\mathrm{CR}$ rates of patients with different subtypes in the two groups were counted. The results showed that the $C R$ rate of $M_{2}$ patients in the observation group was higher than that in the control group $(\mathrm{P}<0.05)$. There was no statistically significant difference in the $C R$ rates of $M_{1}, M_{4}$ and $M_{5}$ patients between the two groups $(\mathrm{P}>0.05)$ (Table III).

Comparison of toxic and side effects. The total incidence of non-hematologic toxic and side effects of patients during and after treatment was compared between the two groups. Patients in the observation group had significantly lower
Table II. Comparison of efficacy between observation and control group [n (\%)].

\begin{tabular}{lcccc}
\hline Groups & no. & CR & PR & OR \\
\hline Observation & 36 & $29(80.56)$ & $2(5.56)$ & $31(86.11)$ \\
Control & 40 & $30(75.00)$ & $4(10.00)$ & $34(85.00)$ \\
t-test & & 0.337 & 0.515 & 0.019 \\
P-value & & 0.562 & 0.473 & 0.891 \\
\hline
\end{tabular}

incidence of cardiotoxicity and alopecia than those in the control group $(\mathrm{P}<0.05)$. The incidence of hematologic BMD 
Table III. Non-hematologic toxic and side effects of observation and control group [n (\%)].

\begin{tabular}{|c|c|c|c|c|}
\hline \multirow{2}{*}{$\begin{array}{l}\text { Toxic and } \\
\text { side effects }\end{array}$} & \multicolumn{2}{|c|}{ Groups } & \multirow[b]{2}{*}{$\chi^{2}$} & \multirow[b]{2}{*}{ P-value } \\
\hline & Observation $(\mathrm{n}=36)$ & Control $(n=40)$ & & \\
\hline Anorexia & $16(44.44)$ & $17(42.50)$ & 0.029 & 0.864 \\
\hline Nausea and vomiting & $12(33.33)$ & $13(32.50)$ & 0.006 & 0.939 \\
\hline Stomatitis & $3(8.33)$ & $5(12.50)$ & 0.350 & 0.555 \\
\hline Fever & $16(44.44)$ & $24(60.00)$ & 1.839 & 0.175 \\
\hline Respiratory system & $28(77.78)$ & $30(75.00)$ & 0.081 & 0.776 \\
\hline Digestive system & $12(33.33)$ & $16(40.00)$ & 0.362 & 0.548 \\
\hline Urinary system & $0(0.00)$ & $1(2.50)$ & 0.912 & 0.340 \\
\hline Rash & $4(11.11)$ & $6(15.00)$ & 0.251 & 0.617 \\
\hline Increased ALT & $2(5.56)$ & $3(7.50)$ & 0.117 & 0.733 \\
\hline Cardiotoxicity & $1(2.78)$ & $8(20.00)$ & 5.383 & 0.020 \\
\hline Alopecia & $4(11.11)$ & $12(30.00)$ & 4.067 & 0.044 \\
\hline
\end{tabular}

Table IV.Comparison of incidence of myelosuppression between two groups [n (\%)].

\begin{tabular}{lcccc}
\hline \multirow{5}{*}{ Grades } & $\begin{array}{c}\text { Groups } \\
(\mathrm{n}=36)\end{array}$ & $\begin{array}{c}\text { Control } \\
(\mathrm{n}=40)\end{array}$ & $\mathrm{t}$ & P-value \\
\cline { 2 - 5 } & $0(0.00)$ & $0(0.00)$ & - & - \\
\hline 0 & $2(5.56)$ & $0(0.00)$ & 2.282 & 0.131 \\
I & $5(13.89)$ & $3(7.50)$ & 0.821 & 0.365 \\
II & $8(22.22)$ & $6(15.00)$ & 0.658 & 0.417 \\
III & $21(58.33)$ & $31(77.50)$ & 4.782 & 0.029 \\
IV & & & & \\
\hline
\end{tabular}

Table V. Comparison of incidence of BMD of patients between two groups [n (\%)].

\begin{tabular}{lcccc}
\hline \multicolumn{5}{c}{ Groups } \\
\cline { 2 - 4 } Grades & $\begin{array}{c}\text { Observation } \\
(\mathrm{n}=36)\end{array}$ & $\begin{array}{c}\text { Control } \\
(\mathrm{n}=40)\end{array}$ & $\mathrm{t}$ & P-value \\
\hline 0 & $0(0.00)$ & $0(0.00)$ & - & - \\
I & $2(5.56)$ & $0(0.00)$ & 2.282 & 0.131 \\
II & $5(13.89)$ & $3(7.50)$ & 0.821 & 0.365 \\
III & $8(22.22)$ & $6(15.00)$ & 0.658 & 0.417 \\
IV & $21(58.33)$ & $31(77.50)$ & 4.782 & 0.029 \\
\hline
\end{tabular}

grading of patients during and after treatment was compared between the two groups. Patients in the observation group had lower incidence of BMD at grade IV than those in the control group, with no statistically significant difference between the two groups $(\mathrm{P}<0.05)$ (Tables IV and V).

Survival analysis. All patients were followed up with a median follow-up time of 16.5-28 months. The median progression-free

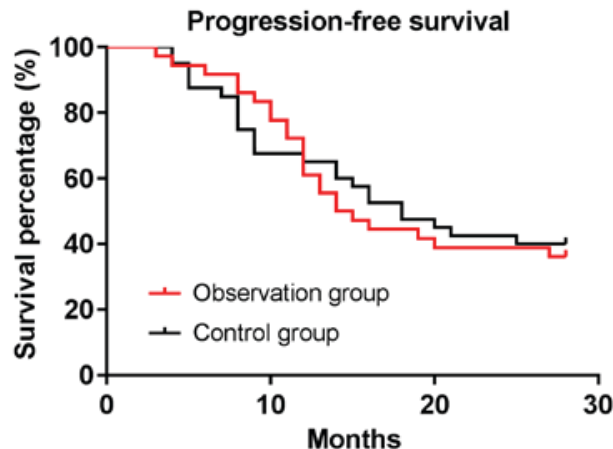

Figure 1. Comparison of disease-free survival between observation and control group. Kaplan-Meier was used for survival analysis. The results showed that the median progression-free survival time of patients was 14.5 months in the observation group and 18 months in the control group. The progression-free survival rate of patients was $36.11 \%$ in the observation group and $40.00 \%$ in the control group, with no difference between the two groups $(\mathrm{P}>0.05)$.

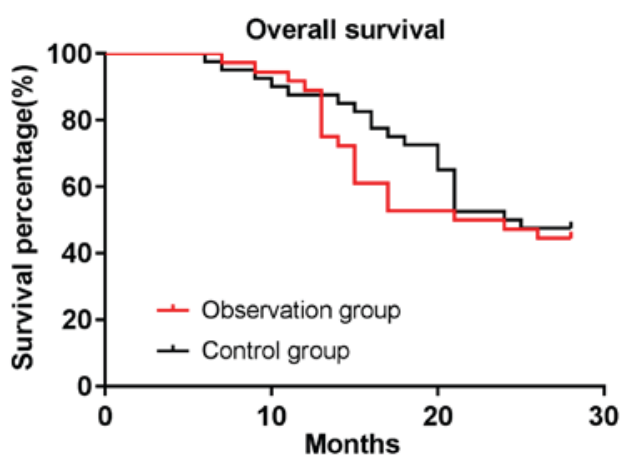

Figure 2. Comparison of survival between observation group and control group. Kaplan-Meier was used for survival analysis. The results showed that the median survival time of patients was 22.5 months in the observation group and 24.5 months in the control group. The OS rate of patients was $44.44 \%$ in the observation group and $47.50 \%$ in the control group, with no statistical difference between the two groups $(\mathrm{P}>0.05)$.

survival time of patients was 14.5 months in the observation group and 18 months in the control group. The progression-free survival rate of patients was $36.11 \%$ in the observation group 
and $40.00 \%$ in the control group, with no difference between the two groups $(\mathrm{P}>0.05)$. The median survival time of patients was 22.5 months in the observation group and 24.5 months in the control group. The OS rate of patients was $44.44 \%$ in the observation group and $47.50 \%$ in the control group, with no difference between the two groups ( $\mathrm{P}>0.05)$ (Figs. 1 and 2).

\section{Discussion}

AML is a hematological malignant tumor with high heterogeneity, which has a higher risk of mortality in adult leukemia (16-18). At present, its pathogenesis is not clear, but smoking and obesity have been confirmed to be its risk factors $(19,20)$. AML occurs mostly in elderly patients whose age is older when diagnosed, and their therapeutic efficacy and tolerance are significantly worse with age (3). In addition, there are often bleeding, infection or other adverse symptoms during the onset and treatment of the disease, which usually means worse prognosis (1). Therefore, the key to the diagnosis and treatment of AML is how to ensure the efficacy on AML patients while finding a safer treatment regimen.

At present, AML is still treated based on anthracyclines combined with cytarabine. In order to consolidate the efficacy, the dose of daunorubicin is often clinically increased to improve therapeutic effects currently, which causes larger toxic and side effects at the same time (21). As described in the previous section, compared to the classic daunorubicin regimen, the chemotherapy regimens of pirarubicin and mitoxantrone combined with cytarabine have better efficacy in the treatment of acute leukemia $(11,12)$. Therefore, in this study, the efficacy and toxic and side effects of these two regimens in the treatment of AML were compared, in order to provide a reference for the treatment of AML.

The results of this study showed that the CR rate of patients was $\geq 75 \%$ and the OR rate was $\geq 85 \%$ in both groups, and there were no statistically significant differences in the CR, $\mathrm{PR}$ and OR rates of patients between the two groups. The progression-free survival rates of patients in the two groups were 36.11 and $40.00 \%$, respectively, and the OS rates were 44.44 and $47.50 \%$, respectively. There was no difference in the progression-free survival rate and OS rate of patients between the two groups. It is shown that the efficacy of pirarubicin combined with cytarabine is similar to that of mitoxantrone combined with cytarabine. Pirarubicin is a semi-synthetic derivative of doxorubicin, which adds tetrahydropyranyl in the original structure. It has been approved for the clinical treatment of gastric cancer, uterine cancer and acute leukemia in Japan (22). Compared to doxorubicin, pirarubicin has faster intracellular uptaking and smaller cardiotoxicity, and is widely used in doxorubicin-resistant cell lines (23). Mitoxantrone has been proved to initiate an effective anti-tumor immune response by apoptosis induction in B16-f1 tumor cells, and the membrane translocation and coating of calreticulin on the surface of apoptotic cells (24). On one hand, in a study by Singh et al (25), mitoxantrone combined with other drugs was found to increase patients' CR rate $(83.3 \%)$ and 1-year OS rate $(81.7 \%)$, which are slightly better than those in our findings (the CR rate was $75.00 \%$ and the OS rate was $47.50 \%$ ). This may be related to the addition of clofarabine in that study. The survival analysis time in this study is significantly longer than that in the previous study, but the survival rate is not comparable. On the other hand, in the multi-center prospective study by Chen et al (26), the chemotherapy regimens of both pirarubicin and mitoxantrone were found to have better efficacy in the treatment of recurrent and refractory AML in adults, and there was no significant difference in survival. This is similar to our findings, but the CR rate in this study is higher than that in the study by Chen et al (26) $(79.00 \% / 55.60 \%)$, which may be related to the inclusion and exclusion criteria in this study. Patients selected in this study were all AML patients, so the CR rate of drugs may be higher. All of the above studies have confirmed our findings directly or indirectly, and both regimens have better efficacy in AML patients.

The CR rate of $\mathrm{M}_{2}$ patients in the observation group was higher than that in the control group in this study, indicating that pirarubicin may be more effective than mitoxantrone in the treatment of $\mathrm{M}_{2}$ AML. According to the Fab criteria, $\mathbf{M}_{2}$ patients refer to AML patients who have 30-89\% of medullary cells in the blood, with promyelocytes and neutrophils accounting for $>10 \%$, and $<20 \%$ of monocytes (27). Studies have shown that AML patients often have chromosomal abnormalities. For instance, there is a $t(8 ; 21)$ translocation in $40-80 \%$ of $\mathrm{M}_{2}$ AML patients who generally have poor clinical efficacy $(28)$. The $t(8 ; 21)$ translocation leads to the production of RUNX 1/ETO fusion protein that maintains the progression of leukemia by promoting cell cycle progression (29). Currently, there is little research on pirarubicin in $\mathrm{M}_{2}$ patients. Findings in this study are likely to be accidental, but it is also possible that pirarubicin improves the efficacy due to the inhibition of the expression of RUNX 1/ETO fusion protein. This is an interesting argument, but its specific mechanism is not discussed in depth in this study because of the limitations of experimental conditions. This aspect will be focused on in subsequent research.

Finally, patients in the observation group had significantly lower incidence of cardiotoxicity and alopecia than those in the control group, and lower incidence of BMD at grade IV than those in the control group. It is indicated that pirarubicin has less toxic and side effects and is safer to use than mitoxantrone. Liu et al (30) also use pirarubicin-based combination chemotherapy regimen to treat AML, and no severe cardiotoxicity case was found during the treatment. Although there are different degrees of BMD, the treatment is not affected with smaller toxic and side effects. This is similar to our findings. It is suggested that pirarubicin-based treatment regimen may have less toxic and side effects and higher safety in AML patients. Mitoxantrone is found to have a higher response rate to AML at a high dose, but it also leads to decrease in EF, increase in Tei index, and significant decrease in GLS and GCS, as well as higher E/E ratio and lower $\mathrm{E} / \mathrm{A}$ ratio, which show that the ventricular diastolic ability is also damaged (31). Other studies found that mitoxantrone also causes leukopenia, erythropenia and thrombocytopenia in blood, and even treatment-related acute leukemia in the treatment of patients with multiple sclerosis (32). Although the study shows that hematological side effects caused by mitoxantrone are transient, 6 patients still withdrew from the study due to leukopenia. The above research facts show that mitoxantrone can produce more significant toxic and side effects. 
There are still shortcomings in this study. First, due to the limited site and environment, the sample size included is small, causing certain impacts on the survival analysis, so it is expected that multi-center sample collection can be carried out. Second, because of limited experimental conditions, there is no way to further explore the mechanism of action of pirarubicin and mitoxantrone regimens in AML, which needs further study by other scholars.

In conclusion, both pirarubicin combined with cytarabine and mitoxantrone combined with cytarabine have satisfactory efficacy on initially treated AML. Compared to the latter, the former has lower toxic and side effects.

\section{Acknowledgements}

Not applicable.

\section{Funding}

No funding was received.

\section{Availability of data and materials}

The datasets used and/or analyzed during the current study are available from the corresponding author on reasonable request.

\section{Authors' contributions}

SL was involved in writing the manuscript. SL and HW helped with analysis of observation indicators. AL and XW collected and analyzed general information of patients and helped with clinical investigation. All authors read and approved the final manuscript.

\section{Ethics approval and consent to participate}

This study was approved by the Ethics Committee of Weifang People's Hospital (Weifang, China). Patients who participated in this research had complete clinical data. The signed informed consents were obtained from the patients or the guardians.

\section{Patient consent for publication}

Not applicable.

\section{Competing interests}

The authors declare that they have no competing interests.

\section{References}

1. Thompson MP, Waters TM, Kaplan EK, McKillop CN and Martin MG: Hospital volume and acute myeloid leukemia mortality in Medicare beneficiaries aged 65 years and older. Blood 128: 872-874, 2016.

2. Guolo F, Minetto P, Clavio M, Miglino M, Lemoli RM and Gobbi M: Intesive fludarabine-high dose cytarabine-idarubicin combination as induction therapy with risk-adapted consolidation may improve treatment efficacy in younger acute myeloid leukemia (AML) patients: Rationales, evidences and future perspectives. Biosci Trends 11: 110-114, 2017.
3. Medeiros BC, Satram-Hoang S, Hurst D, Hoang KQ, Momin F and Reyes C: Big data analysis of treatment patterns and outcomes among elderly acute myeloid leukemia patients in the United States. Ann Hematol 94: 1127-1138, 2015.

4. Saultz JN and Garzon R: Acute myeloid leukemia: A concise review. J Clin Med 5: 33, 2016.

5. Jabo B, Morgan JW, Martinez ME, Ghamsary M and Wieduwilt MJ: Sociodemographic disparities in chemotherapy and hematopoietic cell transplantation utilization among adult acute lymphoblastic and acute myeloid leukemia patients. PLoS One 12: e0174760, 2017.

6. Chen X, Xie H, Wood BL, Walter RB, Pagel JM, Becker PS, Sandhu VK, Abkowitz JL, Appelbaum FR and Estey EH: Relation of clinical response and minimal residual disease and their prognostic impact on outcome in acute myeloid leukemia. J Clin Oncol 33: 1258-1264, 2015.

7. Murphy T and Yee KWL: Cytarabine and daunorubicin for the treatment of acute myeloid leukemia. Expert Opin Pharmacother 18: 1765-1780, 2017.

8. Lubieniecka JM, Graham J, Heffner D, Mottus R, Reid R, Hogge D, Grigliatti TA and Riggs WK: A discovery study of daunorubicin induced cardiotoxicity in a sample of acute myeloid leukemia patients prioritizes P450 oxidoreductase polymorphisms as a potential risk factor. Front Genet 4: 231, 2013.

9. Zheng S, Zhou S, Qiao G, Yang Q, Zhang Z, Lin F, Min D, Tang L, Li H, Sun Y, et al: Pirarubicin-based chemotherapy displayed better clinical outcomes and lower toxicity than did doxorubicin-based chemotherapy in the treatment of non-metastatic extremity osteosarcoma. Am J Cancer Res 5: 411-422, 2014.

10. Damiani RM, Moura DJ, Viau CM, Caceres RA, Henriques JAP and Saffi J: Pathways of cardiac toxicity: Comparison between chemotherapeutic drugs doxorubicin and mitoxantrone. Arch Toxicol 90: 2063-2076, 2016.

11. Kudo K, Kojima S, Tabuchi K, Yabe H, Tawa A, Imaizumi M, Hanada R, Hamamoto K, Kobayashi R, Morimoto A, et al: Prospective study of a pirarubicin, intermediate-dose cytarabine, and etoposide regimen in children with Down syndrome and acute myeloid leukemia: the Japanese Childhood AML Cooperative Study Group. J Clin Oncol 25: 5442-5447, 2007.

12. Wang WJ, Sun AN and Qiu HY: A systematic review of MA versus IA regimen for patients with acute myelogenous leukemia. Zhonghua Xue Ye Xue Za Zhi 32: 869-870, 2011. (In Chinese).

13. Tamamyan G, Kadia T, Ravandi F, Borthakur G, Cortes J, Jabbour E, Daver N, Ohanian M, Kantarjian H and Konopleva M: Frontline treatment of acute myeloid leukemia in adults. Crit Rev Oncol Hematol 110: 20-34, 2017.

14. George TI, Erba HP, Pollyea DA, Abedi M, Roboz GJ, Thompson MA, Fliss A, Swern AS, Nifenecker M, Kiselev P, et al: Current diagnosis patterns for acute myeloid leukemia: Concordance between clinical practice (Connect ${ }^{\circledR}$ Disease Registry) and WHO 2008 recommendations. Leuk Res 55: S106, 2017.

15. Canaani J, Beohou E, Labopin M, Socié G, Huynh A, Volin L, Cornelissen J, Milpied N, Gedde-Dahl T, Deconinck E, et al: Impact of FAB classification on predicting outcome in acute myeloid leukemia, not otherwise specified, patients undergoing allogeneic stem cell transplantation in CR1: An analysis of 1690 patients from the acute leukemia working party of EBMT. Am J Hematol 92: 344-350, 2017.

16. Rosenblat TL, McDevitt MR, Mulford DA, Pandit-Taskar N, Divgi CR, Panageas KS, Heaney ML, Chanel S, Morgenstern A, Sgouros G, et al: Sequential cytarabine and alpha-particle immunotherapy with bismuth-213-lintuzumab (HuM195) for acute myeloid leukemia. Clin Cancer Res 16: 5303-5311, 2010.

17. Luo Y, Li J, Wang Y, Hao X and Qu F: Nimotuzumab combined with chemotherapy as second- or later-line in the treatment of advanced lung squamous cell carcinoma. Zhongguo Fei Ai Za Zhi 19: 665-669, 2016 (In Chinese).

18. Stein EM and Tallman MS: Novel and emerging drugs for acute myeloid leukemia. Curr Cancer Drug Targets 12: 522-530, 2012.

19. Musselman JR, Blair CK, Cerhan JR, Nguyen P, Hirsch B and Ross JA: Risk of adult acute and chronic myeloid leukemia with cigarette smoking and cessation. Cancer Epidemiol 37: 410-416, 2013.

20. Poynter JN, Richardson M, Blair CK, Roesler MA, Hirsch BA, Nguyen P, Cioc A, Warlick E, Cerhan JR and Ross JA: Obesity over the life course and risk of acute myeloid leukemia and myelodysplastic syndromes. Cancer Epidemiol 40: 134-140, 2016. 
21. Portugal R, Lyrio R, Loureiro M, Urago K, Bard J, Borchardt A, Garnica M and Nucci M: Daunorubicin $90 \mathrm{mg} / \mathrm{m}^{2}$ in acute myeloid leukemia induction: Increased toxicity in young patients. Clin Lymphoma Myeloma Leuk 17: 527-531, 2017.

22. Mizutani H, Hotta S, Nishimoto A, Ikemura K, Miyazawa D, Ikeda Y, Maeda T, Yoshikawa M, Hiraku Y and Kawanishi S: Pirarubicin, an anthracycline anticancer agent, induces apoptosis through generation of hydrogen peroxide. Anticancer Res 37 : 6063-6069, 2017.

23. Tsukigawa K, Liao L, Nakamura H, Fang J, Greish K, Otagiri M and Maeda H: Synthesis and therapeutic effect of styrene-maleic acid copolymer-conjugated pirarubicin. Cancer Sci 106: 270-278, 2015.

24. Cao C, Han Y, Ren Y and Wang Y: Mitoxantrone-mediated apoptotic B16-F1 cells induce specific anti-tumor immune response. Cell Mol Immunol 6: 469-475, 2009.

25. Singh GHH, Ip HW, Yip SF, Kho CSB, Lee KKH, Mak V, Lau JSM, Lau CK, Lin S, Wong SMR, et al: Clofarabine, cytarabine and mitoxantrone (CLAM) for relapsed or refractory acute myeloid leukaemia-interim results of a prospective phase 2 study. In: 22nd Congress of the European Haematology Association, Madrid, PB1679, 2017.

26. Chen F, Wang J, Hou M, Zhao H, Yang E, Ran X, Wang M, Yu W, $\mathrm{Xu} \mathrm{R}$, Wang Z, et al: Prospective multicentre study of chemotherapeutic regimen containing pirarubicin on the treatment of relapsed or refractory acute myeloid leukemia in adults. Zhonghua Xue Ye Xue Za Zhi 35: 388-392, 2014.

27. He G, Wang C, Li Q, Tan H, Chen F, Huang Z, Yu B, Zheng L, Zheng R and Liu D: Clinical and laboratory features of seven patients with acute myeloid leukemia (AML)-M2/M3 and elevated myeloblasts and abnormal promyelocytes. Cancer Cell Int 14: 111, 2014
28. Chen L, Luo LF, Lu J, Li L, Liu YF, Wang J, Liu H, Song H, Jiang H, Chen SJ, et al: FTY720 induces apoptosis of $\mathbf{M}_{2}$ subtype acute myeloid leukemia cells by targeting sphingolipid metabolism and increasing endogenous ceramide levels. PLoS One 9: e103033, 2014.

29. Martinez-Soria N, McKenzie L, Draper J, Ptasinska A, Issa H, Potluri S, Blair HJ, Pickin A, Isa A, Chin PS, et al: The oncogenic transcription factor RUNX1/ETO corrupts cell cycle regulation to drive leukemic transformation. Cancer Cell 34: 626-642.e8, 2018 (In Chinese).

30. Liu Y, He P, Cheng X and Zhang M: Long-term outcome of 31 cases of refractory acute promyelocytic leukemia treated with compound realgar natural indigo tablets administered alternately with chemotherapy. Oncol Lett 10: 1184-1190, 2015.

31. Shaikh AY, Suryadevara S, Tripathi A, Ahmed M, Kane JL, Escobar J, Cerny J, Nath R, McManus DD, Shih J, et al: Mitoxantrone-induced cardiotoxicity in acute myeloid leukemia-A velocity vector imaging analysis. Echocardiography 33: 1166-1177, 2016.

32. PastuszakZ,Stepien A,TomczykiewiczKand Piusinska-Macoch R: Blood count in patients with multiple sclerosis treated with mitoxantrone in short time observation. Acta Pol Pharm 73: 1369-1373, 2016

This work is licensed under a Creative Commons Attribution-NonCommercial-NoDerivatives 4.0 International (CC BY-NC-ND 4.0) License. 